\title{
Erratum to: Dihydromyricetin Improves Hypobaric Hypoxia-Induced Memory Impairment via Modulation of SIRT3 Signaling
}

Peng Liu ${ }^{1} \cdot$ Dan Zou ${ }^{1} \cdot$ Ka Chen $^{1} \cdot$ Qicheng Zhou $^{1} \cdot$ Yanxiang Gao ${ }^{1} \cdot$ Yujie Huang ${ }^{1}$.

Jundong Zhu ${ }^{1} \cdot$ Qianyong Zhang ${ }^{1} \cdot$ Mantian $\mathbf{M i}^{1}$

Published online: 16 January 2017

(C) Springer Science+Business Media New York 2017

Erratum to: Mol Neurobiol (2016) Vol (53): 7200-7212

DOIs 10.1007/s12035-015-9627-y

The original version of this article unfortunately contained an error on Figure 2.

The bar graph of Figure $2 \mathbf{g}$ (Quantified results of postsynaptic density in CA1 hippocampal neurons) was the same for the bar graph of Figure 2 b (Quantified results of normal mitochondrial frequency in CA1 hippocampal neurons).

The authors hereby publish the correct Figure 2.

The online version of the original article can be found at http://dx.doi. org/10.1007/s12035-015-9627-y.

Mantian Mi

mimantian@outlook.com

1 Research Center for Nutrition and Food Safety, Institute of Military Preventive Medicine, Third Military Medical University; Chongqing Key Laboratory of Nutrition and Food Safety; Chongqing Medical Nutrition Research Center, Chongqing 400038,

People's Republic of China 
a
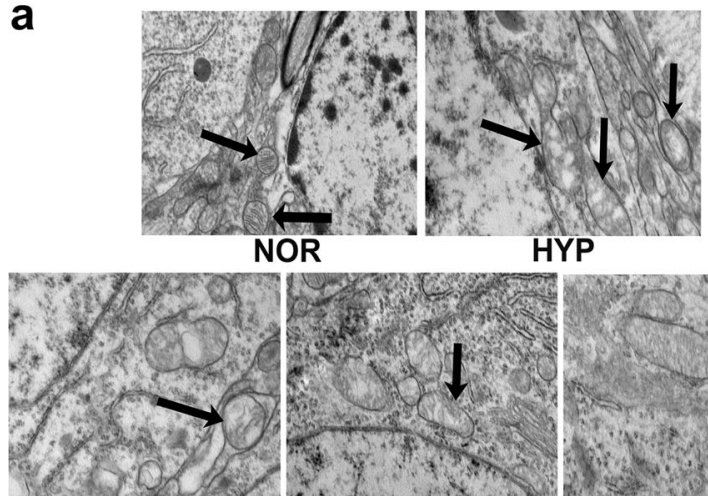

50

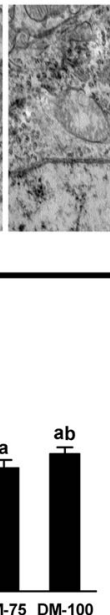

d

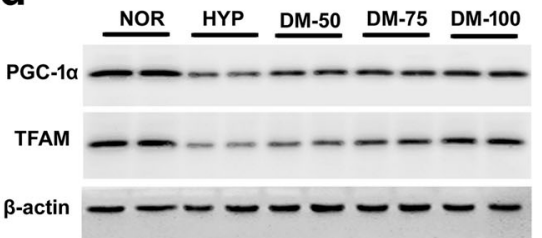

e

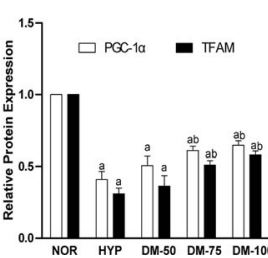

Fig. 2 Effect of DM on hippocampal synaptic structure, mitochondrial structure and related protein expression. a Changes in mitochondrial morphology were examined by TEM. Mitochondria were mainly tubular and with normal structure (black arrows) in the NOR group. Mitochondria with cristae loss (black arrows) were observed in the HYP, DM-50, DM-75 group. b Quantified results of normal mitochondrial frequency in CA1 hippocampal neurons. c Relative mtDNA number in the hippocampus was determined by measuring the level of ND1 relative to that of $\beta$-actin by quantitative PCR $\mathbf{d}$ f

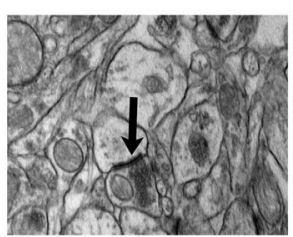

NOR

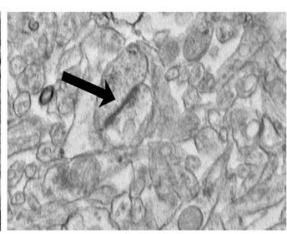

HYP

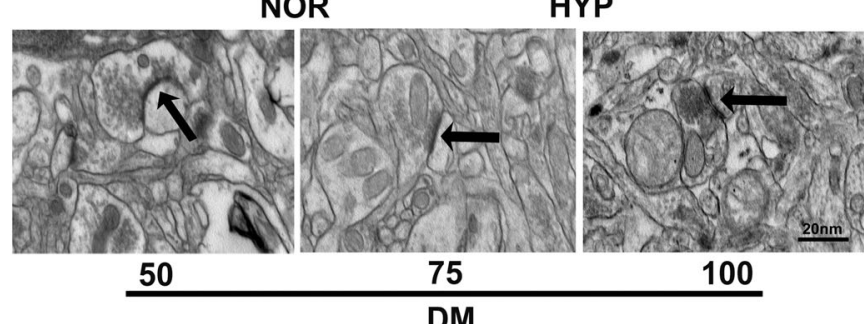

g

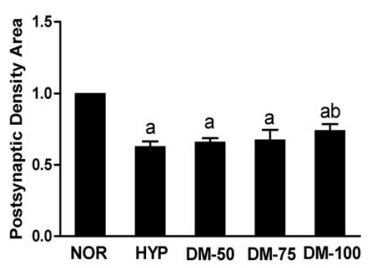

h

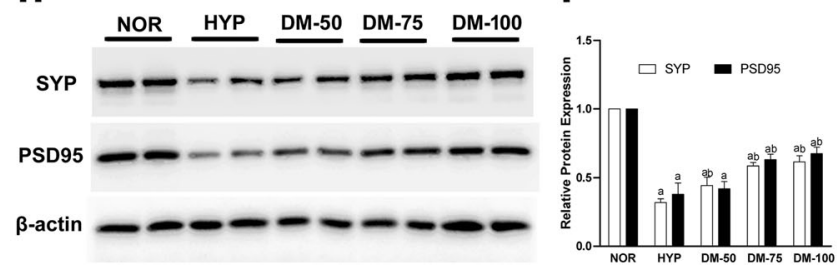

Representative immunoblots of PGC- $1 \alpha$ and TFAM expression; $\beta$-actin was used as a loading control. e Quantitative analysis of PGC- $1 \alpha$ and TFAM expression. f Ultrastructural changes in synaptic structure (black arrows) were examined by TEM. g Quantified results of postsynaptic density in CA1 hippocampal neurons. $\mathbf{h}$ Representative immunoblots of SYP and PSD95 expression; $\beta$-actin was used as a loading control. i Quantitative analysis of SYP and PSD95 expression. Data are expressed as mean $\pm \mathrm{SD}, n=3 .{ }^{\mathrm{a}} P<0.05$ vs. NOR, ${ }^{\mathrm{b}} P<0.05$ vs. HYP. a, f scale bar, $200 \mathrm{~nm}$ 